\title{
Total Domination Number of Generalized Petersen Graphs
}

\author{
Jianxiang CAO ${ }^{1}$, Weiguo $\mathrm{LIN}^{2}$, Minyong $\mathrm{SHI}^{3}$ \\ ${ }^{1}$ School of Computer Science, Communication University of China, Beijing 100024, China \\ ${ }^{2}$ School of Computer Science, Communication University of China, Beijing 100024, China \\ ${ }^{3}$ School of Animation, Communication University of China, Beijing 100024, China
}

\begin{abstract}
Generalized Petersen graphs are an important class of commonly used interconnection networks and have been studied. The total domination number of generalized Petersen graphs $\mathrm{P}(\mathrm{m}, 2)$ is obtained in this paper.
\end{abstract}

Keywords: generalized Petersen graphs, total domination set, total domination number, regular graph, domination set, domination number

\section{廣義 Petersen 圖的全控制數}

\author{
曹建香 ${ }^{1}$, 林衛國 ${ }^{2}$, 石民勇 $^{3}$ \\ ${ }^{1}$ 中國傳媒大學計算機學院，北京 $100024 ;{ }^{2}$ 中國傳媒大學計算機學院，北京 100024 ; \\ ${ }^{3}$ 中國傳媒大學動畫學院，北京 100024
}

\begin{abstract}
摘 要: 廣義 Petersen 圖是一類重要的並被廣泛研究的互聯網絡拓撲結構, 控制數是圖論中的一個重要參 數，而全控制數是控制數的一個變形，本文確定了廣義 Petersen 圖的全控制數。
\end{abstract}

關鍵詞：廣義 Petersen 圖, 全控制集, 全控制數, 正則圖, 控制集, 控制數

\section{1. 引言}

互聯網路具有廣泛的含義, 泛指元件集合與通信 通道集合按照一定的點對點方式相互連接所形成的系 統。網路中的元件和元件之間的連接方式成為該網路 的拓撲結構, 人們通常把網路中的元件抽象成一個 點, 把通信通道抽象成兩點之間的連線, 該網路中的 拓撲結構就被抽象成一個圖。例如, 一個通信網路可 以用圖做模型, 網路中的站點作為圖中的點, 站點之 間的連接作為圖中的邊。出於通信的需要, 人們在站 點安置發射器, 利用某個站點上的發射器, 可以把資 訊發送到所有與該站相鄰的站點。為了覆蓋整個網路 必須適當選取一些站點來放置發射器, 使得每個站點 都能接收到來自這些發射器的信號。在這裏抽象成圖
論問題就是站點的選取相當於尋找圖中的控制集, 出 於經濟的考虑, 發射器越少越好, 這就是要去找圖中 的最小控制集, 所需要發射器的數目就是圖中的控制 數。

上面提到的概念僅僅是控制這個概念的最原始的 形式，在現實問題中很多問題都與控制的概念有關 係，根據實際問題的不同，對控制的概念做了不同的 變形, 這裏要介紹的一種是一全控制數。然而對任 意的圖確定其全控制數是 NP-完全問題, 所以一般只 能確定某些特殊圖類的全控制數, 如在[1]中研究了兩 類特殊圖廣義 De Bruijn 和廣義 Kauta 的全控制數。這 裏我們主要求解廣義 Petersen 圖的全控制數。廣義 Petersen 圖是 Petersen 圖的推廣, 是一類重要的互聯網 
路, 因而受到廣泛的研究, 在[2]中研究了廣義 Petersen 圖的 Hamiltonian 圈, 在[3]中研究了廣義 Petersen 圖 的交叉數 ( crossing number), 在 [4]中給出了廣義 Petersen 圖 $\mathrm{P}(\mathrm{m}, 2)$ 的直徑和寬直徑, 在[5][6]中求出了 廣義 Petersen 圖的控制數的一個上界。本文求出了廣 義 Petersen 圖的全控制數。

\section{2. 基本概念}

所謂圖(graph)指的是一個二元組 $(\mathrm{V}, \mathrm{E})$, 其中 $\mathrm{V}$ 是圖的頂點集(vertex-set), 它的元素稱為圖的頂點 (vertex), 而 $\mathrm{E}$ 是圖的邊集(edge-set), 它的元素稱為圖 的邊(edge), 一般用 $V(G)$ 和 $E(G)$ 來表示圖 $G$ 的頂點集 和邊集, 以區別於其他的圖。一條邊 $\mathrm{e}$ 和兩個頂點相 關聯(incident), 即 $\mathrm{e}=(\mathrm{u}, \mathrm{v})$, 其中 $\mathrm{u}, \mathrm{v} \in \mathrm{V}$, 此時稱 $\mathrm{u}, \mathrm{v}$ 為 $\mathrm{e}$ 的端點(end-vertex), 並稱 $\mathrm{u}, \mathrm{v}$ 是相鄰的(adjacent)。 直觀地看, 頂點就是空間中的點, 而邊就是兩點之間 的連接. 若這樣的連接没有方向, 即對圖中每條邊都有 $(\mathrm{u}, \mathrm{v})=(\mathrm{v}, \mathrm{u})$, 那麼稱這樣的圖為無向圖 (undirected graph), 其中的邊稱為無向邊, 並可用 $\mathrm{u} v$ 這種形式來 表示 若 $\mathrm{W} \subseteq \mathrm{V}(\mathrm{G})$, 則 $\mathrm{W}$ 在 $\mathrm{G}$ 中的導出子圖記為 $<\mathrm{W}>$, 設 $\mathrm{v} \in \mathrm{V}(\mathrm{G})$, 令 $\mathrm{N}(\mathrm{v})=\{\mathrm{u} \in \mathrm{V}(\mathrm{G}) \mid \mathrm{uv} \in \mathrm{E}(\mathrm{G})\}$ 和 $N[v]=\{v\} \cup N(v)$; 設 $A \subseteq V(G)$, 令 $N[A]=\{v \mid v \in A$ 或 $\exists \mathrm{u} \in \mathrm{A}$ 使 $\mathrm{uv} \in \mathrm{E}(\mathrm{G})\}$; 其中與頂點 $\mathrm{u}$ 相鄰接的頂點數 稱為 $\mathrm{u}$ 的度, 若一個圖中各個頂點的度都是 $\mathrm{k}$, 則稱 該圖為 $\mathrm{k}$ 正則圖。

定義 1 廣義 Petersen 圖, 記為 $\mathrm{P}(\mathrm{m}, \mathrm{a})$, 其定義如 下: $\mathrm{P}(\mathrm{m}, \mathrm{a})$ 的頂點集為 $\mathrm{U} \cup \mathrm{W}$, 其中 $\mathrm{U}=\left\{\mathrm{u}_{1}, \mathrm{u}_{2}, \ldots, \mathrm{u}_{\mathrm{m}}\right\}$, $\mathrm{W}=\left\{\mathrm{w}_{1}, \mathrm{~W}_{2}, \ldots, \mathrm{w}_{\mathrm{m}}\right\} ; \quad \mathrm{P}(\mathrm{m}, \mathrm{a})$ 的邊集為 $\left(\mathrm{u}_{\mathrm{i}}\right.$, $\left.w_{i}\right)(1 \leq i \leq m),\left(u_{i}, u_{i+1}\right)\left(i \geq 1, u_{i}=u_{j}\right.$, 如果 $\left.i \equiv j(\bmod m)\right)$ 和 $\left(w_{i}\right.$, $\left.w_{i+a}\right)\left(i \geq 1, w_{i}=w_{j}\right.$, 如果 $\left.i \equiv j(\bmod m)\right)$ 。稱 $U$ 中的點為外圈 點, $\mathrm{W}$ 中的點為內圈點。廣義 Petersen 圖是 Coxter 於 1951 年引入的, 顯然 Petersen 圖即為 $\mathrm{P}(5,2)$ 。

定義 2 若 $(u, v)$ 是圖 $G$ 中的一條邊, 則稱點 $u$ 全 控制點 $\mathrm{v}$ 。如果 $\mathrm{T} \subseteq \mathrm{V}(\mathrm{G})$ 能夠全控制所有 $\mathrm{G}$ 的頂點, 則稱 $\mathrm{T}$ 是 $\mathrm{G}$ 的全控制集。所含頂點最少的全控制集稱 為最小全控制集, 其中的頂點數稱為全控制數, 記作 $\gamma_{\mathrm{t}}(\mathrm{G})$ 。

不難看出, 全控制集就是不含孤立點的控制集, 並 且對任意圖, 全控制集也肯定是控制集。文中所討論的

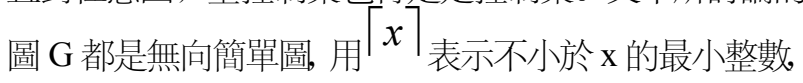
文中其他未加說明的概念和術語均參見文獻[7]。

\section{3. 主要結論}

為了證明主要定理, 先給出一個引理。

引理 1 若 $\mathrm{G}$ 是一個具有 $\mathrm{n}$ 個頂點的 $\mathrm{k}$ 正則圖, 則 $\gamma_{\mathrm{t}}(\mathrm{G}) \geq\left\lceil\frac{n}{k}\right\rceil$ 。

證明: 由全控制數的定義知道, 每個頂點可以控 制除自身之外的 $\mathrm{k}$ 個頂點, 若設 $\mathrm{T}$ 是 $\mathrm{G}$ 的最小全控制 集, 設 $u, v \in T$, 且 $u v$ 是一條邊, 在最好的情況下, $\mathrm{N}(\mathrm{u}) \cap \mathrm{N}(\mathrm{v})=\phi$, 則此時 $\mathrm{T}$ 中每個點恰好控制了 $\mathrm{k}$ 個 點, 故 $\mathrm{T}$ 中頂點數不小於 $\left\lceil\frac{n}{k}\right\rceil$ 。

定理 1 廣義 Petersen 圖 $\mathrm{P}(\mathrm{m}, 2)$ 的全控制數為:

$$
\gamma_{\mathrm{t}}(\mathrm{P}(\mathrm{m}, 2))=\left\{\begin{array}{cc}
2 \mathrm{k} & m=3 k \\
2(k+1) & m=3 k+1 \\
2(k+1) & m=3 k+2
\end{array}\right.
$$

中 $\mathrm{k} \geq 2$ 。

證明: 證明過程主要是找出 $\mathrm{P}(\mathrm{m}, 2)$ 的一個最小全 控制集。下麵根據廣義 Petersen 圖的結構分三種情況 討論:

情形 1 當 $\mathrm{m}=3 \mathrm{k}$ 時, 證 $\gamma_{\mathrm{t}}(\mathrm{P}(3 \mathrm{k}, 2))=2 \mathrm{k}, \mathrm{k}=1,2, \ldots$

取 $\mathrm{T}=\left\{\mathrm{u}_{1}, \mathrm{w}_{1}, \mathrm{u}_{4}, \mathrm{w}_{4}, \ldots, \mathrm{u}_{3 \mathrm{k}-2}, \mathrm{w}_{3 \mathrm{k}-2}\right\},|\mathrm{T}|=2 \mathrm{k}$, 下面分兩 種情形來證明 $\mathrm{T}$ 就是 $\mathrm{P}(3 \mathrm{k}, 2)$ 的一個最小全控制集。

子情形 1.1 當 $\mathrm{k}$ 為偶數時

此時 $\mathrm{P}(3 \mathrm{k}, 2)$ 的內圈點構成了兩個長度為 $3 \mathrm{k} / 2$ 的 內圈, 外圈點構成一個長度為 $3 \mathrm{k}$ 的外圈, 記外圈 $\mathrm{C}=\mathrm{u}_{1} \mathrm{u}_{2} \mathrm{u}_{3} \ldots \mathrm{u}_{3 \mathrm{k}} \mathrm{u}_{1}$ 。兩個內圈分別用 $\mathrm{C}_{1}, \mathrm{C}_{2}$ 來表示:

$$
\begin{aligned}
& \mathrm{C}_{1}=\mathrm{w}_{1} \mathrm{~W}_{3} \mathrm{~W}_{5} \ldots \mathrm{w}_{3 \mathrm{k}-1} \mathrm{~W}_{1} ; \\
& \mathrm{C}_{2}=\mathrm{W}_{2} \mathrm{~W}_{4} \mathrm{~W}_{6} \ldots \mathrm{w}_{3 \mathrm{k}} \mathrm{W}_{2} .
\end{aligned}
$$

因為 $\left(u_{i}, w_{i}\right)(1 \leq i \leq m)$ 是 $P(3 k, 2)$ 中的邊, 所以 $T$ 中的 頂點已被全控制。同時 $\mathrm{T}$ 中的外圈點 $\mathrm{u}_{3 \mathrm{i}-2}(1 \leq \mathrm{i} \leq \mathrm{k})$ 全控 制了外圈上的點 $\mathrm{u}_{3 \mathrm{k}}, \mathrm{u}_{2}, \mathrm{u}_{3 \mathrm{i}-3}, \mathrm{u}_{3 \mathrm{i}-1}$, 其中 $2 \leq \mathrm{i} \leq \mathrm{k}$, 這些點 及 $\mathrm{T}$ 中的外圈點恰好是全部外圈上的點, 即外圈上的 點全部被全控制。

對於 $\mathrm{T}$ 中內圈 $\mathrm{C}_{1}$ 上的點 $\mathrm{w}_{1}, \mathrm{w}_{7}, \ldots, \mathrm{w}_{3 \mathrm{k}-5}$ 全控制了 $\mathrm{C}_{1}$ 上的 $\mathrm{w}_{3 \mathrm{k}-1}, \mathrm{w}_{3}, \mathrm{w}_{5}, \ldots, \mathrm{w}_{3 \mathrm{k}-4}$ 。 類似的對於 $\mathrm{T}$ 中內圈 $\mathrm{C}_{2}$ 上的點 $\mathrm{W}_{4}, \mathrm{~W}_{10}, \ldots, \mathrm{w}_{3 \mathrm{k}-2}$ 全控制了 $\mathrm{C}_{2}$ 上的 $\mathrm{w}_{2}, \mathrm{w}_{6}, \mathrm{~W}_{8}$, $\mathrm{w}_{10}, \ldots, \mathrm{w}_{3 \mathrm{k}}$ 。因此 $\mathrm{T}$ 是一個全控制集, 故 $\gamma_{\mathrm{t}}(\mathrm{P}(3 \mathrm{k}, 2)) \leq|\mathrm{T}|=2 \mathrm{k}$ 。

又因為 $\mathrm{P}(3 \mathrm{k}, 2)$ 是一個 3 正則圖, 由引理 1 知, $\gamma_{\mathrm{t}}(\mathrm{P}(3 \mathrm{k}, 2)) \geq(2 \times 3 \mathrm{k}) / 3=2 \mathrm{k}$ 。綜上可得 $\gamma_{\mathrm{t}}(\mathrm{P}(3 \mathrm{k}, 2))=2 \mathrm{k}$ 。 
子情形 1.2 當 $\mathrm{k}$ 為奇數時

此時 $\mathrm{P}(3 \mathrm{k}, 2)$ 的外圈點跟子情形 1.1 一樣, 用 $\mathrm{C}$ 來 表示, 內圈點構成了一個長度為 $3 \mathrm{k}$ 的內圈, 記為 $\mathrm{C}_{1}$ $=\mathrm{W}_{1} \mathrm{~W}_{3} \mathrm{~W}_{5} \ldots \mathrm{W}_{3 \mathrm{k}} \mathrm{W}_{2} \mathrm{~W}_{4} \mathrm{~W}_{6} \ldots \mathrm{W}_{3 \mathrm{k}-1} \mathrm{~W}_{1}$. 類似子情形 1.1 , 易知 $\mathrm{T}$ 中的外圈點 $\mathrm{u}_{3 \mathrm{i}-2}(1 \leq \mathrm{i} \leq \mathrm{k})$ 全控制了外圈上的點 $\mathrm{u}_{3 \mathrm{k}}, \mathrm{u}_{2}$, $u_{3 i-3}, u_{3 i-1}$, 其中 $2 \leq i \leq k$, 這些點及 $T$ 中的外圈點恰好是 全部外圈上的點，即外圈上的點全部被全控制。

對於 $\mathrm{T}$ 中內圈 $\mathrm{C}_{1}$ 上的點 $\mathrm{w}_{1}, \mathrm{w}_{4}, \ldots, \mathrm{W}_{3 \mathrm{k}-2}$ 全控制了 $\mathrm{C}_{1}$ 上的 $\mathrm{w}_{3 \mathrm{k}}, \mathrm{W}_{3}, \mathrm{w}_{5}, \ldots, \mathrm{W}_{3 \mathrm{k}}$. 被全控制。因此無論內圈點 還是外全點都可由 $\mathrm{T}$ 中的點全控制, 即 $\mathrm{T}$ 是一個全控 制集，故 $\gamma_{\mathrm{t}}(\mathrm{P}(3 \mathrm{k}, 2)) \leq|\mathrm{T}|=2 \mathrm{k}$ 。

又因為 $\mathrm{P}(3 \mathrm{k}, 2)$ 是一個 3 正則圖, 由引理 1 知, $\gamma_{\mathrm{t}}$ $(\mathrm{P}(3 \mathrm{k}, 2)) \geq(2 \times 3 \mathrm{k}) / 3=2 \mathrm{k}$ 。綜上可得 $\gamma_{\mathrm{t}}(\mathrm{P}(3 \mathrm{k}, 2))=2 \mathrm{k}$ 。

綜合子情形 1.1 和 1.2 可知, 當 $\mathrm{m}=3 \mathrm{k}, \mathrm{k} \geq 1$ 時, 有 $\gamma_{\mathrm{t}}(\mathrm{P}(3 \mathrm{k}, 2))=2 \mathrm{k}$ 。證畢!

情形 2 當 $\mathrm{m}=3 \mathrm{k}+1$ 時,證 $\gamma_{\mathrm{t}}(\mathrm{P}(3 \mathrm{k}+1,2))=2(\mathrm{k}+1)$, $\mathrm{k}=1,2, \ldots \circ$

下麵分三種情形來討論:

子情形 1.1 當 $k=1$ 時, 此時 $\mathrm{P}(4,2)$ 的結構比較特 殊, 只有外圈, 没有內圈, 頂點集 $\mathrm{W}=\left\{\mathrm{w}_{1}, \mathrm{w}_{2}, \mathrm{w}_{3}, \mathrm{w}_{4}\right\}$ 構成的子圖只有兩點邊 $\left(\mathrm{w}_{1}, \mathrm{w}_{3}\right)$ 和 $\left(\mathrm{w}_{2}, \mathrm{w}_{4}\right)$ 。假設 $\mathrm{T}$ 是 $\mathrm{P}(4,2)$ 的最小全控制集, 又由於 $\mathrm{P}(4,2)$ 是 3 正則圖, 故 由引理 1 知 $\gamma_{\mathrm{t}}(\mathrm{P}(4,2)) \geq\left[\frac{2 \times 4}{3}\right]=3$ 。若 $\gamma_{\mathrm{t}}(\mathrm{P}(4,2))=3$, 即 $|\mathrm{T}|=3$, 由全控制的定義知, $\mathrm{T}$ 的導出子圖不能含有 孤立點, 故 $\mathrm{T}$ 中的 3 點構成的子圖必須是連通的, 分 析 $\mathrm{P}(4,2)$ 的結構知道, 這樣的 3 個點生成的子圖中有 兩條邊, 下麵分 4 種情況來討論:

(1) 若該 3 點是外圈上的點, 則 $\mathrm{W}$ 中的除與該 3 點相鄰的剩下的一個點不能被全控制;

(2) 若 $\mathrm{T}$ 中該 3 點中有 2 個是外圈上的點，另一 個是內圈上的點, 不失一般性, 不妨設 $\mathrm{T}=\left\{\mathrm{u}_{1}, \mathrm{u}_{2}, \mathrm{w}_{1}\right\}$, 則此時 $\mathrm{w}_{4}$ 不被全控制;

（3）若 $\mathrm{T}$ 中有 1 個點是外圈點, 另 2 個點為 $\mathrm{W}$ 中 的點, 不失一般性, 設 $\mathrm{T}=\left\{\mathrm{u}_{1}, \mathrm{w}_{1}, \mathrm{w}_{3}\right\}$, 則, 此時有 $\mathrm{w}_{2}, \mathrm{w}_{4}$ 不被全控制;

(4) $\mathrm{T}$ 中的 3 個點不可能為 $\mathrm{W}$ 中的點, 因為 $\mathrm{W}=\left\{\mathrm{W}_{1}, \mathrm{~W}_{2}, \mathrm{~W}_{3}, \mathrm{~W}_{4}\right\}$ 導出的子圖中只有兩條邊, 該子圖是 不連通的, 所以不存在 3 個點構成的連通子圖。

由上面 4 種情況的討論可知, $\mathrm{P}(4,2)$ 不可能由 3 個點全控制, 而存在四個點 $\mathrm{w}_{1}, \mathrm{w}_{2}, \mathrm{w}_{3}$ 及 $\mathrm{w}_{4}$ 可全控制
$\mathrm{P}(4,2)$ 。故 $\gamma_{\mathrm{t}}(\mathrm{P}(4,2))=4=2(\mathrm{k}+1)$ 。

當 $\mathrm{k} \geq 2$ 時, 此時分兩種情況來討論, $\mathrm{k}$ 為偶數和 $\mathrm{k}$ 為奇數。

\section{子情形 1.2 當 $\mathrm{k}$ 為偶數時}

此時 $\mathrm{P}(3 \mathrm{k}+1,2)$ 有一個外圈 $\mathrm{C}$ 和一個內圈 $\mathrm{C}_{1}$, 分 別為

$\mathrm{C}=\mathrm{u}_{1} \mathrm{u}_{2} \mathrm{u}_{3} \ldots \mathrm{u}_{3 \mathrm{k}} \mathrm{u}_{1}$

$\mathrm{C} 1=\mathrm{w}_{1} \mathrm{~W}_{3} \mathrm{~W}_{5} \ldots \mathrm{w}_{3 \mathrm{k}+1} \mathrm{~W}_{2} \mathrm{~W}_{4} \mathrm{~W}_{6} \ldots \mathrm{w}_{3 \mathrm{k}} \mathrm{W}_{1}$;

因為 $\mathrm{P}(3 \mathrm{k}+1,2)$ 是一個 3 正則圖, 故由引理 1 得 $\gamma_{\mathrm{t}}$ $(\mathrm{P}(3 \mathrm{k}+1,2)) \geq\left\lceil\frac{2(3 k+1)}{3}\right\rceil=2 \mathrm{k}+1$ 。

現令 $\mathrm{T}_{1}=\left\{\mathrm{u}_{1}, \mathrm{w}_{1}, \mathrm{u}_{4}, \mathrm{w}_{4}, \ldots, \mathrm{u}_{3 \mathrm{k}-2}, \mathrm{w}_{3 \mathrm{k}-2}, \mathrm{u}_{3 \mathrm{k}-1}, \mathrm{u}_{3 \mathrm{k}+1}\right\}$, 此 時 $T_{1}$ 中的點 $u_{i}$ 與 $w_{i}$ 相互全控制, 且 $u_{1}$ 全控制了 $u_{3 k+1}$ 和 $u_{2}, u_{3 k+1}$ 全控制了 $u_{3 k}$ 其他的 $T_{1}$ 中的 $u_{i}$ 全控制了 $u_{i-1}$ 和 $u_{i+1}$, 這裏 $i=4,7, \ldots, 3 k-2 ;$ 類似地, $w_{1}$ 全控制了 $w_{3 k}, w_{3}$, $\mathrm{T}_{1}$ 中的 $\mathrm{w}_{\mathrm{i}}$ 全控制了 $\mathrm{w}_{\mathrm{i}-2}$ 和 $\mathrm{w}_{\mathrm{i}+2}$ ，這裏 $\mathrm{i}=4,7, \ldots, 3 \mathrm{k}-2$, 同時 $u_{3 k-1}$ 和 $u_{3 k+1}$ 分別全控制了 $w_{3 k-1}$ 和 $w_{3 k+1}$ 。即 $T_{1}$ 是 $\mathrm{P}(3 \mathrm{k}+1,2)$ 的一個全控制集, 而 $\left|\mathrm{T}_{1}\right|=2(\mathrm{k}+1)$, 故 $\gamma_{\mathrm{t}}$ $(\mathrm{P}(3 \mathrm{k}+1,2)) \leq 2(\mathrm{k}+1)$, 結 合 $\gamma_{\mathrm{t}}$ $(\mathrm{P}(3 \mathrm{k}+1,2)) \geq\left\lceil\frac{2(3 k+1)}{3}\right\rceil=2 \mathrm{k}+1$ 。可得 $2 \mathrm{k}+1 \leq \gamma_{\mathrm{t}}$ $(\mathrm{P}(3 \mathrm{k}+1,2)) \leq 2(\mathrm{k}+1)$ 。

下麵來證明 $\gamma_{\mathrm{t}} \quad(\mathrm{P}(3 \mathrm{k}+1,2)) \neq 2 \mathrm{k}+1$, 從而得出 $\gamma_{\mathrm{t}}$ $(\mathrm{P}(3 \mathrm{k}+1,2))=2(\mathrm{k}+1)$ 。

反證法, 假設 $\gamma_{\mathrm{t}}(\mathrm{P}(3 \mathrm{k}+1,2))=2 \mathrm{k}+1$ 。則 $\exists$ 一個最 小全控制集 $\mathrm{T}$, 滿足 $|\mathrm{T}|=2 \mathrm{k}+1$ 。

因為 $\mathrm{T}$ 是最小全控制集, 所以 $\mathrm{T}$ 中没有孤立頂 點, 由廣義 Petersen 圖的結構性質知, $\mathrm{T}$ 中至少存在 3 個頂點, 這 3 個頂點導出的子圖是一條路, 這 3 個頂 點要麼都是相鄰的外圈點或內圈點, 要麼 2 個點是外 圈點一個點是內圈點或反之。若 3 個點都是內圈點或 者外圈點, 不失一般性, 不妨設該 3 點為 $\mathrm{w}_{3}, \mathrm{w}_{5}, \mathrm{w}_{7}$, 這 3 個點相互全控制並且控制了外圈點 $\mathrm{u}_{3}, \mathrm{u}_{5}, \mathrm{u}_{7}$ 和內圈 點 $\mathrm{w}_{1}, \mathrm{w}_{9}$, 此時外圈上, $\mathrm{u}_{3}, \mathrm{u}_{4}, \mathrm{u}_{5}, \mathrm{u}_{6}, \mathrm{u}_{7}$ 是連續相鄰的 點, 但 $\mathrm{u}_{4}, \mathrm{u}_{6}$ 未被控制, 故要麼由內圈上的 $\mathrm{w}_{4}, \mathrm{w}_{6}$ 來 全控制, 要麼至少由 $\mathrm{u}_{5}$ 來全控制, 若是前者, 即 $\mathrm{w}_{3}, \mathrm{w}_{5}$, $\mathrm{w}_{7}, \mathrm{w}_{4}, \mathrm{w}_{6}$ 共控制了 14 個頂點, 故剩下的 $2(3 \mathrm{k}+1)-14$ 個頂點, 至少需要 $2(3 \mathrm{k}+1)-14 / 3=2 \mathrm{k}-4$ 個點來全控制, 此時滿足這個最少數的條件是 $\mathrm{T}$ 中其他的任 2 個點都 没有公共鄰接點, 但是在 $\mathrm{w}_{11}, \ldots, \mathrm{w}_{3 \mathrm{k}+1}$ 之間共有 $(3 \mathrm{k}-8) / 2$ 個點未被全控制，要使這些點被全控制，其中不能有 
相鄰的點在 $\mathrm{T}$ 中, 否則這樣兩個相鄰的點控制了外圈 上間隔為 1 的點, 要使這個間隔點被全控制必然導致 有兩個 T 中點有公共鄰接點, 這與 2k-4 個全控制點矛 盾。因此要使 $\mathrm{w}_{11}, \ldots, \mathrm{w}_{3 \mathrm{k}+1}$ 被控制, $\mathrm{T}$ 中控制這些點的 相鄰點只能一個在外圈上一個在內圈上, 但這至少需 要 $(3 \mathrm{k}-8) /(2 \times 3)$ 個點, 顯然這也導致 T 中有 2 個全控制 點有共同的鄰域。所以這 3 個點其中有 2 個是外圈 ( 或 內圈 ) 點另一個點是內圈 (或外圈) 點, 不失一般性, 不妨設這 3 個點為 $\mathrm{u}_{3}, \mathrm{w}_{3}, \mathrm{w}_{5}$, 這 3 個點全控制了 $\mathrm{u}_{2}, \mathrm{u}_{3}$, $\mathrm{u}_{4}, \mathrm{w}_{1}, \mathrm{w}_{3}, \mathrm{w}_{5}, \mathrm{w}_{7}$, 這樣還有 $3 \mathrm{k}+1-3+3 \mathrm{k}+1-4=6 \mathrm{k}-5$ 未被 全控制, 又因為每個點度為 3 只能全控制 3 個點, 故這 $6 \mathrm{k}-5$ 個點至少需要 2k-1 個點來控制, 這樣全控制點至 少有 $2 \mathrm{k}-1+3=2 \mathrm{k}+2$ 這與 $|\mathrm{T}|=2 \mathrm{k}+1$ 矛盾。綜上所述可 知, 不存在 $2 \mathrm{k}+1$ 個點全控制 $\mathrm{P}(3 \mathrm{k}+1,2)$ 。故 $\gamma_{\mathrm{t}}$ $(\mathrm{P}(3 \mathrm{k}+1,2))=2(\mathrm{k}+1)$ 。

子情形 1.3 當 $\mathrm{k}$ 為奇數時

在此種情況下有兩個內圈, 證明類似子情形 1.2 , 這裏不再討論。

綜合上面的情況可知故 $\gamma_{\mathrm{t}}(\mathrm{P}(3 \mathrm{k}+1,2))=2(\mathrm{k}+1)$, 情形 2 證里!

情形 3 當 $m=3 k+2$ 時, $k \geq 1$

此時, 由引理 1 可得 $\gamma_{\mathrm{t}}(\mathrm{P}(3 \mathrm{k}+2,2)) \geq$ $\left\lceil\frac{2(3 k+2)}{3}\right\rceil=2 \mathrm{k}+2$ 。

要證明 $\gamma_{\mathrm{t}}(\mathrm{P}(3 \mathrm{k}+2,2))=2 \mathrm{k}+2$, 只要證明存在一個全 控制集 $T \subseteq V(P(3 k+2,2))$, 且 $|T|=2 k+2$ 即可。

子情形 1.1 當 $\mathrm{k}$ 為偶數時

此時類似前面可知 $\mathrm{P}(3 \mathrm{k}+2,2)$ 的外圈點構成一個 長度為 $3 \mathrm{k}+2$ 的外圈, 記外圈 $\mathrm{C}=\mathrm{u}_{1} \mathrm{u}_{2} \mathrm{u}_{3} \ldots \mathrm{u}_{3 \mathrm{k}+2} \mathrm{u}_{1}$ 。 $\mathrm{P}(3 \mathrm{k}+2,2)$ 的內圈點構成了兩個長度為 $3 \mathrm{k} / 2$ 的內圈, 兩 個內圈分別用 $\mathrm{C}_{1}, \mathrm{C}_{2}$ 來表示:

$$
\mathrm{C}_{1}=\mathrm{w}_{1} \mathrm{~W}_{3} \mathrm{~W}_{5} \ldots \mathrm{W}_{3 \mathrm{k}+1} \mathrm{~W}_{1} \text {; }
$$$$
\mathrm{C}_{2}=\mathrm{w}_{2} \mathrm{~W}_{4} \mathrm{~W}_{6} \ldots \mathrm{w}_{3 \mathrm{k}+2} \mathrm{~W}_{2} \text {. }
$$

取 $T=\left\{u_{2}, w_{2}, u_{5}, w_{5}, \ldots, u_{3 k-1}, w_{3 k-1}, u_{3 k}, u_{1}\right\}$, 顯然 $T$ 中的點 $u_{i}$ 和 $w_{i}$ 相互全控制, 這裏 $i=2,5, \ldots, 3 k-1$. 且 $u_{i}$ 全控制了 $\mathrm{u}_{\mathrm{i}-1}$ 和 $\mathrm{u}_{\mathrm{i}+1}, \mathrm{u}_{3 \mathrm{k}}$ 全控制了 $\mathrm{u}_{3 \mathrm{k}+1}, \mathrm{u}_{1}$ 全控制了 $\mathrm{u}_{3 \mathrm{k}+2}$, 即外圈上的點可由 $\mathrm{T}$ 中的點全控制。由於 $\mathrm{T}$ 中 的點 $\mathrm{w}_{3 i+2}$ 全控制了內圈 $\mathrm{C}_{1}$ 上的 $\mathrm{w}_{3 \mathrm{i}}$ 和 $\mathrm{w}_{3 \mathrm{i}+4}$, 這裏 $\mathrm{i}=1,3, \ldots, \mathrm{k}-1$, 而 $\mathrm{u}_{1}$ 又控制了 $\mathrm{w}_{1}$, 故內圈 $\mathrm{C}_{1}$ 上的點可
由 $\mathrm{T}$ 中的點全控制。對於內圈 $\mathrm{C}_{2}, \mathrm{~T}$ 中的點 $\mathrm{w}_{2}$ 全控 制了內圈 $\mathrm{C}_{2}$ 上的 $\mathrm{w}_{3 \mathrm{k}+2}$ 和 $\mathrm{w}_{4}, \mathrm{~T}$ 中的點 $\mathrm{w}_{3 \mathrm{i}+2}$ 全控制了 內圈 $\mathrm{C}_{2}$ 上的 $\mathrm{w}_{3 \mathrm{i}}$ 和 $\mathrm{w}_{3 \mathrm{i}+4}$, 這裏 $\mathrm{i}=2,4, \ldots, \mathrm{k}-2$, 而 $\mathrm{u}_{3 \mathrm{k}}$ 又 控制了 $\mathrm{w}_{3 \mathrm{k}}$, 故內圈 $\mathrm{C}_{2}$ 上的點也可由 $\mathrm{T}$ 中的點全控 制。綜上所述 $\mathrm{T}$ 是 $\mathrm{P}(3 \mathrm{k}+2,2)$ 的一個全控制集, 並且因 為 $|\mathrm{T}|=2 \mathrm{k}+2$ 。故 $\mathrm{T}$ 是最小控制集。所以 $\gamma_{\mathrm{t}}(\mathrm{P}(3 \mathrm{k}+2,2))=$ $2(\mathrm{k}+1)$ 。

子情形 1.2 當 $\mathrm{k}$ 為奇數時。

此時 $\mathrm{P}(3 \mathrm{k}+2,2)$ 的外圈仍記為 $\mathrm{C}=\mathrm{u}_{1} \mathrm{u}_{2} \mathrm{u}_{3} \ldots \mathrm{u}_{3 \mathrm{k}+2} \mathrm{u}_{1}$, 內圈點只構成了一個長度為 $3 \mathrm{k}$ 的內圈, 記為 $\mathrm{C}_{1}$ $=\mathrm{w}_{1} \mathrm{~W}_{3} \mathrm{~W}_{5} \ldots \mathrm{w}_{3 \mathrm{k}+2} \mathrm{~W}_{2} \mathrm{~W}_{4} \mathrm{~W}_{6} \ldots \mathrm{w}_{3 \mathrm{k}+1} \mathrm{~W}_{1}$ 。

取 $\mathrm{T}=\left\{\mathrm{u}_{2}, \mathrm{w}_{2}, \mathrm{u}_{5}, \mathrm{w}_{5}, \ldots, \mathrm{u}_{3 \mathrm{k}-1}, \mathrm{w}_{3 \mathrm{k}-1}, \mathrm{u}_{3 \mathrm{k}}, \mathrm{u}_{1}\right\}$, 類似子 情形 1.1 可證 $\mathrm{T}$ 是 $\mathrm{P}(3 \mathrm{k}+2,2)$ 最小控制集。所以 $\gamma_{\mathrm{t}}$ $(\mathrm{P}(3 \mathrm{k}+2,2))=2(\mathrm{k}+1)$ 。

綜合這兩種情形, 得到當 $\mathrm{m}=3 \mathrm{k}+2$ 時, $\gamma_{\mathrm{t}}$ $(\mathrm{P}(\mathrm{m}, 2))=2(\mathrm{k}+1)$ 。證里!

\section{REFERENCES}

[1] Huang Jia, On Domination-Stability of Graphs. The doctoral dissertation of the Univerisity of Science and Technology og China.(in Chinese) (黃佳, 圖的控制穩定性研究. 中國科技大學博士論文, 2007).

[2] Bnaani K. Hamiltonian cycles in generalized Petersen graphs [J]. J Combinatorial Theory, 1978, 181-183.

[3] Exoo G, Harary F, Kabell J. The crossing numbers of some generalized Petersen graphs [J]. Math Scand, 1981, 184-188.

[4] Hou Xin-min, Wang Tian-ming. Wide diameters of generalized Petersen graphs [J]. Journal of Mathematical Research and Exposition, 2004, 24(2): 249-253.

[5] QI Deng-ji, Domination Number of Generalized Petersen Graphs ( $\mathrm{m}$ is odd), Journal of Qingdao University of Science and Technology, Vol . 26 No. 1 Apr . 2005, 92-94(in Chinese) (齊登 記. 廣義 Petersen 圖的控制數——當 $\mathrm{m}$ 是奇數時 $\mathrm{P}(\mathrm{m}, 2)$ 的控 制數 [J]. 青岛科技大學學報（自然科學版），2005，26(1): 92-94).

[6] QI Deng-ji, Domination Number of Generalized Petersen Graphs ( $\mathrm{m}$ is even), Journal of Qingdao University of Science and Technology, Vol . 26 No. 2 Apr . 2005, 181-183(in Chinese)(齊 登記. 廣義 Petersen 圖的控制數——當 $\mathrm{m}$ 是偶數時 $\mathrm{P}(\mathrm{m}, 2)$ 的 控制數 [J]. 青島科技大學學報（自然科學版），2005，26(2): 181-183).

[7] Bondy J A, Murty U S R. Graph theory with applications [M]. New York: North-Holland, 1976. 